\title{
Ellipsometrically determined optical properties of nickel-containing tungsten oxide thin films: Nanostructure inferred from effective medium theory
}

Iryna Valyukh, S V Green, C G Granqvist, K Gunnarsson, Hans Arwin and G A Niklasson

\author{
Linköping University Post Print
}

N.B.: When citing this work, cite the original article.

Original Publication:

Iryna Valyukh, S V Green, C G Granqvist, K Gunnarsson, Hans Arwin and G A Niklasson, Ellipsometrically determined optical properties of nickel-containing tungsten oxide thin films: Nanostructure inferred from effective medium theory, 2012, Journal of Applied Physics, (112), 4, 044308.

http://dx.doi.org/10.1063/1.4748166

Copyright: American Institute of Physics (AIP)

http://www.aip.org/

Postprint available at: Linköping University Electronic Press

http://urn.kb.se/resolve?urn=urn:nbn:se:liu:diva- 84352 


\title{
Ellipsometrically determined optical properties of nickel-containing tungsten oxide thin films: Nanostructure inferred from effective medium theory
}

\author{
I. Valyukh, ${ }^{1,2, a)}$ S. V. Green, ${ }^{2}$ C. G. Granqvist, ${ }^{2}$ K. Gunnarsson, ${ }^{2}$ H. Arwin, ${ }^{1}$ \\ and G. A. Niklasson ${ }^{2}$ \\ ${ }^{1}$ Laboratory of Applied Optics, Department of Physics, Chemistry and Biology, Linköping University, \\ SE-58183 Linköping, Sweden \\ ${ }^{2}$ Department of Engineering Sciences, The Angström Laboratory, Uppsala University, P.O. Box 534, \\ SE-75121 Uppsala, Sweden
}

(Received 29 May 2012; accepted 20 July 2012; published online 29 August 2012)

\begin{abstract}
Films of $\mathrm{Ni}_{x} \mathrm{~W}_{1-x}$ oxide with $0.05 \leq x \leq 0.53$ were produced by reactive dc magnetron co-sputtering onto $\mathrm{Si}$. Such films have documented electrochromism. Spectroscopic ellipsometry was used to extract accurate data on the dielectric function in the photon range 0.062 to $5.62 \mathrm{eV}$. The results for 0.62 to $5.62 \mathrm{eV}$ were compared with computations from the Bruggeman effective medium theory applied to two nanostructural models: one representing a random mixture of structural entities characterized by the dielectric functions of $\mathrm{WO}_{3}$ and $\mathrm{NiWO}_{4}$ and the other describing a random mixture of $\mathrm{WO}_{3}$ and $\mathrm{NiO}$. Unambiguous evidence was found in favor of the former model, and hence the films are composed of nanosized tungsten oxide and nickel tungstate. This agrees excellently with an earlier investigation of ours on $\mathrm{Ni}_{x} \mathrm{~W}_{1-x}$ oxide films, where nanostructure was inferred from Raman spectroscopy, x-ray photoelectron spectroscopy, and x-ray diffraction. (C) 2012 American Institute of Physics. [http://dx.doi.org/10.1063/1.4748166]
\end{abstract}

\section{INTRODUCTION}

Electrochromic devices allow their optical transmittance to be electrically modulated and have important applications in energy efficient and comfort enhancing smart windows for buildings as well as for several other applications. ${ }^{1-3}$ Devices based on nickel oxide and tungsten oxide have been found to exhibit excellent performance, ${ }^{4}$ and binary nickel-tungsten oxides have been seen to yield properties that are superior to those of the pure oxides both at the tungsten-rich ${ }^{5,6}$ and at the nickel-rich ${ }^{7-12}$ ends of the compositional range. Furthermore, $\mathrm{NiWO}_{4}$ is known as an electrochromic material. ${ }^{13}$

We have therefore embarked on a comprehensive investigation of the full $\mathrm{Ni}_{x} \mathrm{~W}_{1-x}$ oxide system, with $0 \leq x \leq 1$, and previously reported on the compatibility of these oxides with different electrolytes, ${ }^{14}$ optical properties as determined by spectroscopic ellipsometry, ${ }^{15,16}$ as well as nanostructures and compositions recorded by Raman spectroscopy, x-ray photoelectron spectroscopy, x-ray diffractometry, and Rutherford backscattering spectrometry. ${ }^{17}$ Furthermore, we have studied the electrochromism for films with $0 \leq x \leq 0.6 .^{6}$ In the present paper, we determine the optical properties of $\mathrm{Ni}_{x} \mathrm{~W}_{1-x}$ oxide films by spectroscopic ellipsometry, interpret these properties within effective medium theory, and show that the data are fully consistent with the nanostructural information reported before, thereby lending further credence to a two-phase model with $\mathrm{WO}_{3}$ and $\mathrm{NiWO}_{4}$ as the discrete structural entities for $0 \leq x \leq 0.5^{17}$

\footnotetext{
a) Author to whom correspondence should be addressed. Electronic mail: iryva@ifm.liu.se.
}

\section{THIN FILMS: PREPARATION AND SURFACE CHARACTERIZATION}

Thin films of $\mathrm{Ni}_{x} \mathrm{~W}_{1-x}$ oxide were prepared by reactive dc magnetron co-sputtering from pure tungsten and nickel metal targets onto unheated silicon substrates with native oxide. Different compositions were obtained by varying the power delivered to the individual targets. The nickel fraction $x$ was determined by Rutherford backscattering spectroscopy and $\mathrm{x}$-ray photoelectron spectroscopy. Further details of thin film deposition and of structural and compositional characterization can be found elsewhere. ${ }^{17}$

Surface morphology and roughness, which are relevant for the ellipsometric analysis, were recorded by atomic force microscopy (AFM) using a Veeco Dimension 3100 instrument in tapping mode in air. We used a tip with a radius of $12 \mathrm{~nm}$, which gave a lateral resolution of roughly $30 \mathrm{~nm}$. Figure 1 displays the surface of a $\mathrm{Ni}_{x} \mathrm{~W}_{1-x}$ oxide film with $x \approx 0.13$ and shows a maximum peak-to-valley vertical distance of about $10 \mathrm{~nm}$.

\section{ELLIPSOMETRY}

\section{A. Equipment and techniques}

Optical properties of $\mathrm{Ni}_{x} \mathrm{~W}_{1-x}$ oxide films were recorded with spectroscopic ellipsometry in the photon energy range of $0.062 \leq E \leq 5.62 \mathrm{eV}$. Measurements in the ultravioletvisible-near infrared (UV-VIS-NIR) region at $0.62 \leq E$ $\leq 5.62 \mathrm{eV}$ were performed using a variable-angle ellipsometer with rotating analyzer (VASE, J. A. Woollam Co., Inc.) in steps of $0.02 \mathrm{eV}$. An infrared (IR) Fourier transform rotating compensator ellipsometer (J. A. Woollam Co., Inc.) was employed for measurements in the IR range at 500 to $5000 \mathrm{~cm}^{-1}$ wavenumbers $(0.062 \leq E \leq 0.620 \mathrm{eV})$ with a 

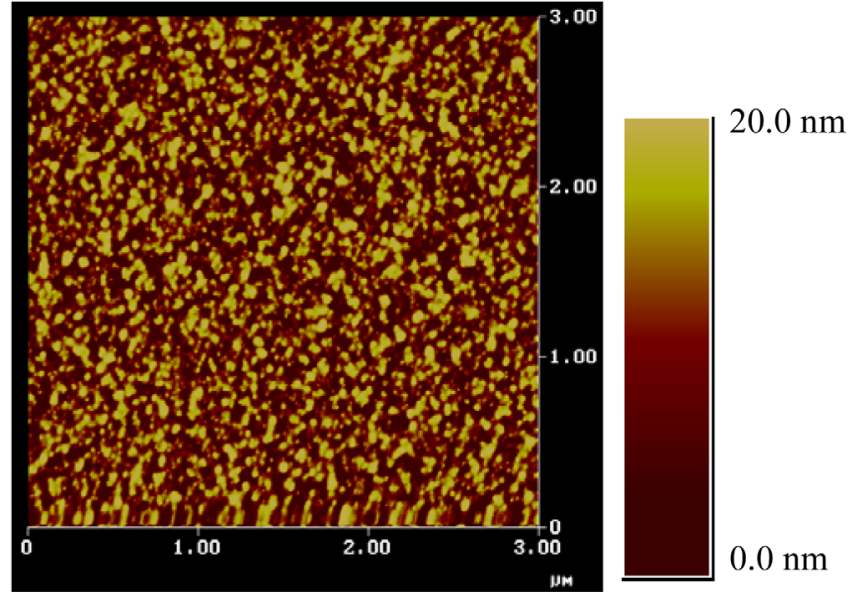

FIG. 1. AFM micrograph for a $\mathrm{Ni}_{x} \mathrm{~W}_{1-x}$ oxide film with $x \approx 0.13$. Protrusion heights are indicated by color, as shown in the right-hand part.

resolution of $4 \mathrm{~cm}^{-1}$. Incidence angles in the $50^{\circ}$ to $70^{\circ}$ range were used in steps of $5^{\circ}$ and $10^{\circ}$ for the UV-VIS-NIR and IR regions, respectively, to provide data with good signal-to-noise ratio at each photon energy. Modeling and regression analysis were performed with WVASE32 ${ }^{\mathcal{C}}$ software (J. A. Woollam Co., Inc.).

The complex dielectric function, denoted $\varepsilon(E)=\varepsilon_{1}(E)$ $+i \varepsilon_{2}(E)$, was obtained from the measurements of the change in the polarization state of light due to its interaction with the

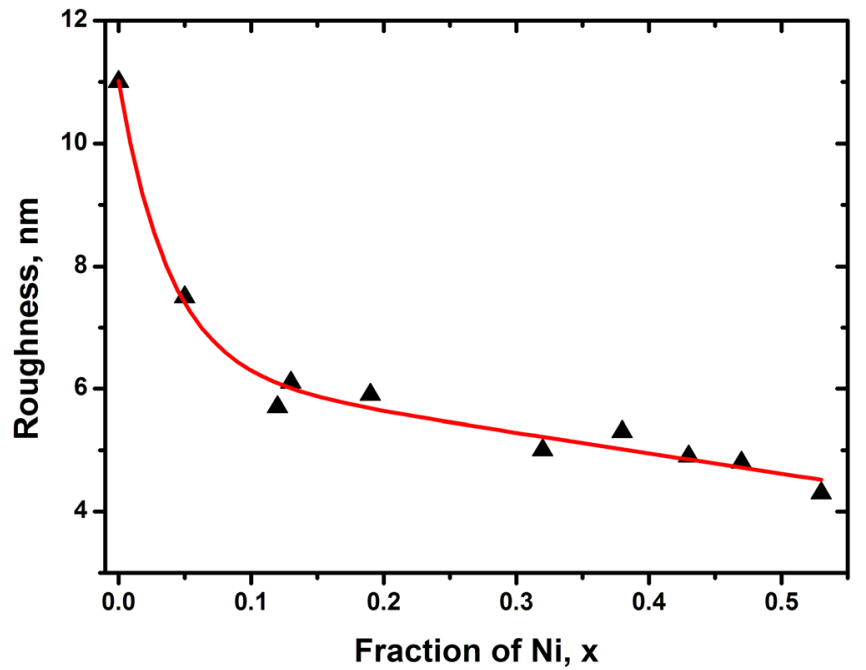

FIG. 2. Ellipsometrically determined roughness of $\mathrm{Ni}_{x} \mathrm{~W}_{1-x}$ oxide thin films $v s \mathrm{Ni}$ fraction $x$. The curve was drawn for convenience.

thin film. This change is described by the fundamental ellipsometric equation ${ }^{18}$

$$
\rho *=\frac{R_{p}}{R_{s}}=\tan \Psi \exp (i \Delta),
$$

where $\rho^{*}$ is the complex-valued ratio between the reflection coefficients $R_{p}$ and $R_{s}$ for $p$ and $s$ polarized light, respectively
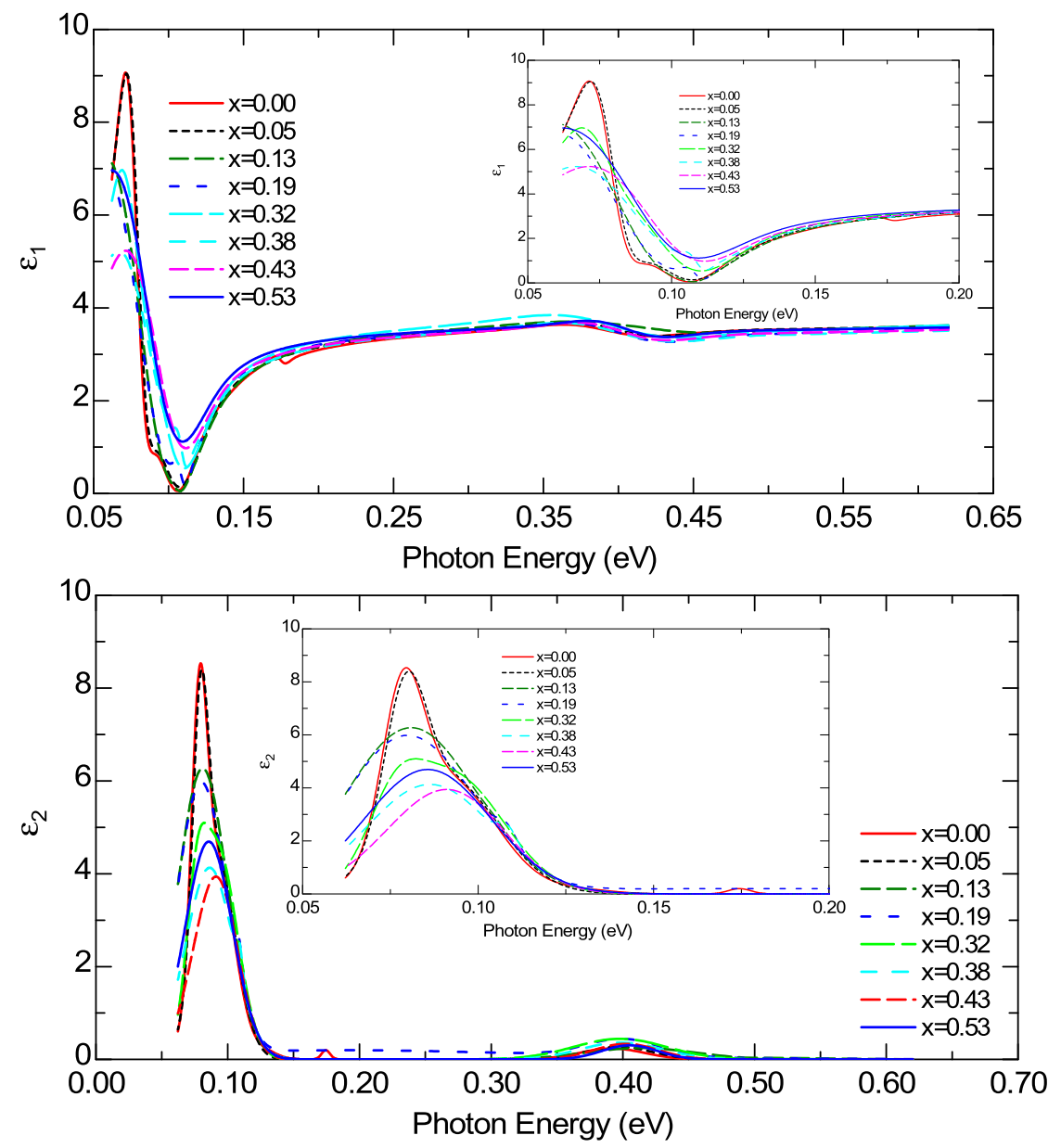

FIG. 3. Real ( $\varepsilon_{1}$, upper panel) and imaginary $\left(\varepsilon_{2}\right.$, lower panel) parts of the complex dielectric functions of $\mathrm{Ni}_{x} \mathrm{~W}_{1-x}$ oxide films, with the shown compositions $x$, as determined from spectroscopic ellipsometry with a parametric model in the 0.062 $\leq E \leq 0.62 \mathrm{eV}$ range of photon energy $E$. Insets show the same spectra for $0.062 \leq E \leq 0.20 \mathrm{eV}$. 
(i.e., polarization of the electric field parallel and perpendicular to the plane of incidence), and $\Psi$ and $\Delta$ are the experimentally determined parameters. An optical model for each film was created and used to calculate $R_{p}$ and $R_{s}$ and corresponding values of $\Psi$ and $\Delta$. The best fit of the free parameters in the model was found by using a Levenberg-Marquardt regression algorithm for minimization of the mean square error between the model data and the experimental results. ${ }^{18}$ In this way, the model dielectric function parameters, film thickness, roughness, and other material parameters can be extracted from the experiment.

The film roughness, shown in Fig. 1, was accounted for in the optical model by representing a surface layer as an effective medium (cf. Sec. IV below) with $50 \%$ voids; this improved the fitting of the ellipsometric data significantly. A structural model with air-roughness-film- $\mathrm{SiO}_{z}-$ substrate thus was used in order to describe the $\mathrm{Ni}_{x} \mathrm{~W}_{1-x}$ oxide films on Si. A thickness non-uniformity parameter, allowing for an in-plane variation of the layer thickness, was also included. The optical properties of the substrate and of the native silicon oxide were taken from the literature. ${ }^{19}$ A measurement on a reference sample showed that the thickness of the $\mathrm{SiO}_{z}$ layer was $2.9 \pm 0.1 \mathrm{~nm}$, which was used in the further data analysis.

A parametric model, which consists of a set of oscillators, was used to represent the dielectric function of the $\mathrm{Ni}_{x} \mathrm{~W}_{1-x}$ oxide films. The contribution to $\varepsilon$ due to electronic transitions in the UV region was approximated by a TaucLorentz oscillator $\varepsilon_{T L},{ }^{20}$ and Kramers-Kronig-correct functions $\varepsilon_{G}$ with Gaussian broadening ${ }^{21}$ were used to model $\varepsilon$ near the band gap at high $\mathrm{Ni}$ fractions. The contribution to $\varepsilon$ due to lattice vibrations in the IR was accounted for by two to four Gaussian oscillators. ${ }^{21}$

The total model dielectric function used for the $\mathrm{Ni}_{x} \mathrm{~W}_{1-x}$ oxide films then has the form

$$
\varepsilon=\varepsilon_{\infty}+\varepsilon_{T L}+\sum_{i} \varepsilon_{G i},
$$

where $\varepsilon_{\infty}$ is the high-frequency contribution modeled with a pole in the UV region. ${ }^{18} \mathrm{~A}$ detailed description of the parametric model used to represent the $\mathrm{Ni}_{x} \mathrm{~W}_{1-x}$ oxide films is given elsewhere. ${ }^{16}$

\section{B. Data}

The analysis leads to an ellipsometrically determined roughness of the $\mathrm{Ni}_{x} \mathrm{~W}_{1-x}$ oxide films as shown in Fig. 2. It is seen that the roughness drops monotonically as the $\mathrm{Ni}$ fraction is increased. Roughness data obtained by AFM and ellipsometry in general show a quantitative and linear relation $^{22,23}$ and the results in Fig. 2 are in line with the magnitude of the AFM-determined protrusion height shown in Fig. 1. Detailed comparison of the roughness data obtained by AFM and ellipsometry is not within the scope of this paper.

The ellipsometric data in the UV-VIS-NIR and IR ranges were analyzed using the parametric model in Eq. (2). Film thicknesses lay between 124 and $304 \mathrm{~nm}$, with thickness non-uniformities around $4 \% .{ }^{16} \mathrm{We}$ obtained results on
$\varepsilon_{1}(E)$ and $\varepsilon_{2}(E)$ for $\mathrm{Ni}_{x} \mathrm{~W}_{1-x}$ oxide films with $0 \leq x \leq 0.53$ as shown in Figs. 3 and 4. It should be noted that some correlations appear between the parameters of the Gaussian oscillators, which implies that the solutions are not unique and there exist multiple sets of Gaussians which provide the same dielectric functions. However, in this investigation the final dielectric functions rather than the model parameter values are of main interest, so the fact that multiple parameter sets can produce nearly identical dielectric functions, which are uniquely determined by ellipsometry, is not a concern. The pronounced features in the IR are due to lattice vibrations, mainly $\mathrm{W}-\mathrm{O}$ modes below $0.1 \mathrm{eV}$ and a vibration mode centered at $\sim 0.4 \mathrm{eV}$ and probably due to bound water. ${ }^{1}$ A pronounced absorption peak is seen in the UV; it shifts to higher energy as $x$ is increased.

\section{EFFECTIVE MEDIUM ANALYSES AND IMPLICATIONS FOR THE NANOSTRUCTURE}

\section{A. Techniques}

Our earlier work ${ }^{17}$ showed that $\mathrm{Ni}_{x} \mathrm{~W}_{1-x}$ oxide films with $0 \leq x \leq 0.5$ could be described as nanocomposites comprised $\mathrm{WO}_{3}$ and $\mathrm{NiWO}_{4}$. We now consider whether the optical data presented above can be understood from this premise by turning to effective medium theory. ${ }^{3,24-26} \mathrm{We}$ first assume that the two components of the nanocomposite can be described by the complex dielectric functions for $x=0$ (corresponding to $\mathrm{WO}_{3}$ ) and $x=0.53$ (approximately corresponding to $\mathrm{NiWO}_{4}$ ); this is referred to as the $\mathrm{WO}_{3}-$ $\mathrm{NiWO}_{4}$ model below. We further assume that the structural
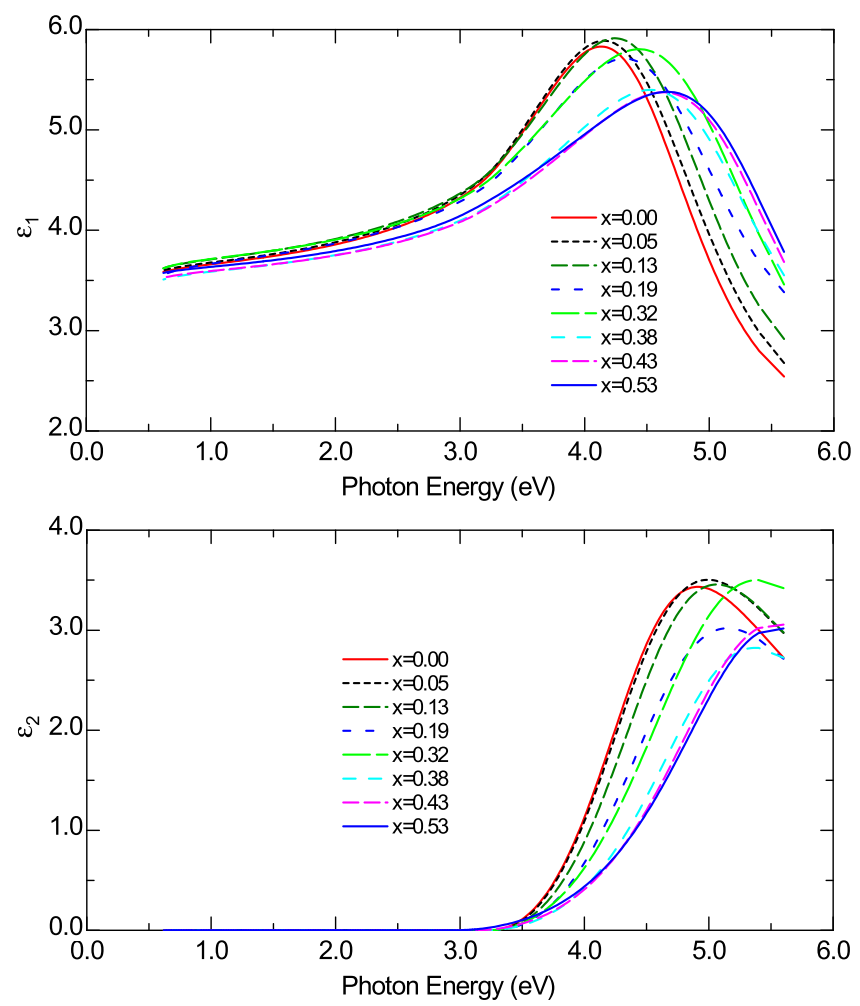

FIG. 4. Real ( $\varepsilon_{1}$, upper panel) and imaginary ( $\varepsilon_{2}$, lower panel) parts of the complex dielectric functions of $\mathrm{Ni}_{x} \mathrm{~W}_{1-x}$ oxide films, with the shown compositions $x$, as determined from spectroscopic ellipsometry with a parametric model in the $0.62 \leq E \leq 5.62 \mathrm{eV}$ range of photon energy $E$. 
TABLE I. Fraction of $\mathrm{Ni}$, given as $x$ in $\mathrm{Ni}_{x} \mathrm{~W}_{1-x}$ oxide, and volume fractions $f$ for $\mathrm{NiWO}_{4}$ and $\mathrm{NiO}$ of the two nanostructural models.

\begin{tabular}{llllllll}
\hline \hline$x$ & 0.05 & 0.13 & 0.19 & 0.32 & 0.38 & 0.43 & 0.53 \\
\hline$f_{\mathrm{NiWO}_{4}}$ & 0.07 & 0.19 & 0.29 & 0.54 & 0.68 & 0.90 & 1.00 \\
$f_{\mathrm{NiO}}$ & 0.02 & 0.07 & 0.09 & 0.17 & 0.19 & 0.25 & 0.33 \\
\hline \hline
\end{tabular}

entities are small enough that the measured optical properties can be represented by an effective dielectric function, which is an average over the dielectric functions of the components. The averaging should be done in different ways depending on the nanotopology of the composite material. ${ }^{27}$ Here, we presume that the composite consists of a random mixture of the components, in which case the Bruggeman theory ${ }^{28}$ is appropriate. An alternative theory might be the Maxwell Garnett theory, ${ }^{29}$ which pertains to discrete particles of one
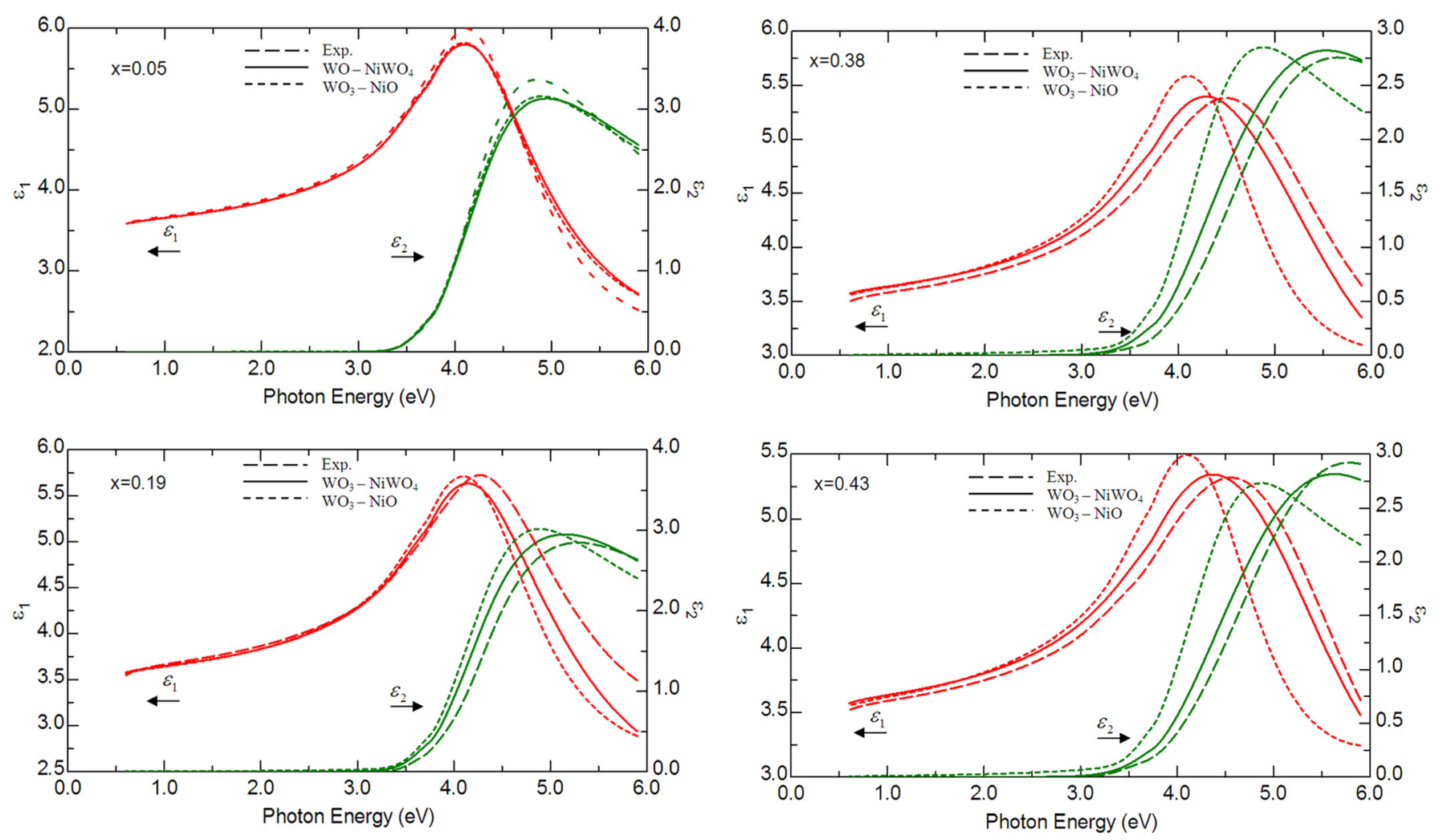

phase embedded in a continuous host of the other phase. The two effective medium theories coincide if the fraction of one of the phases is small.

We also considered a second nanostructural model with a random mixture of tungsten oxide and nickel oxide (whose optical properties were determined by ellipsometry in an earlier paper of ours ${ }^{15}$ ); this is referred to as the $\mathrm{WO}_{3}-\mathrm{NiO}$ model. By carrying out effective-medium-based calculations analogous to those for a composite of $\mathrm{WO}_{3}$ and $\mathrm{NiWO}_{4}$, it will be possible to test whether the latter structural model is indeed superior or whether a successful fitting to experimental data may be spurious.

The Bruggeman theory gives effective dielectric functions $\varepsilon^{B R}$ by

$$
\left(1-f_{m}\right) \frac{\varepsilon_{W O_{3}}-\varepsilon^{B R}}{\varepsilon_{W O_{3}}+2 \varepsilon^{B R}}+f_{m} \frac{\varepsilon_{m}-\varepsilon^{B R}}{\varepsilon_{m}+2 \varepsilon^{B R}}=0,
$$

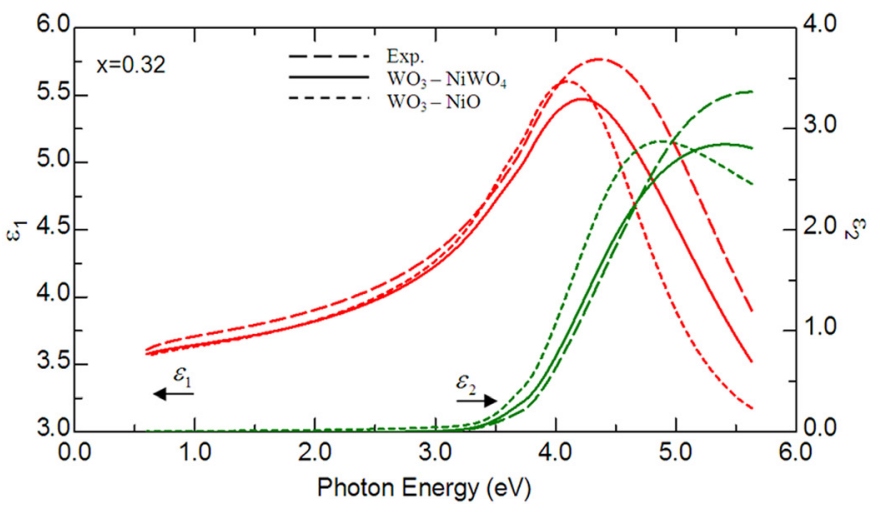

FIG. 5. Spectral complex dielectric function, $\varepsilon_{1}+i \varepsilon_{2}$ with pertinent vertical axes indicated by arrows, for $\mathrm{Ni}_{x} \mathrm{~W}_{1-x}$ oxide films having the shown compositions $x$. Experimental data are shown together with calculations based on two nanostructural models, as shown in the figure. Experimental data were reported also in Fig. 4. 
where $f_{m}$ is the volume fraction of $\mathrm{NiWO}_{4}$ or $\mathrm{NiO}$ and $\varepsilon_{m}$ is the dielectric function of $\mathrm{NiWO}_{4}$ or NiO. The volume fractions appropriate for the two models are readily obtained from

$$
\begin{aligned}
& f_{\mathrm{NiWO}_{4}}=\frac{1}{1+\frac{1-2 x}{x} \frac{M_{\mathrm{WO}_{3}}}{M_{N i O_{4}}} \frac{\rho_{\mathrm{NWO}_{4}}}{\rho_{W O_{3}}},}, \\
& f_{N i O}=\frac{1}{1+\frac{1-x}{x} \frac{M_{W O_{3}}}{M_{N i O}} \frac{\rho_{N i O}}{\rho_{W O_{3}}},},
\end{aligned}
$$

where $M$ is molar mass and $\rho$ is density.

Estimations of $\rho$ are fraught with some uncertainty, and bulk values are not useful since the films were deposited under conditions that render them porous, and electrochromic films of tungsten oxide and nickel oxide can have densities that are as low as $\sim 60 \%$ of those of corresponding bulk materials. ${ }^{1}$ However, our earlier work on $\mathrm{Ni}_{x} \mathrm{~W}_{1-x}$ oxide films with $0 \leq x \leq 1$ used Rutherford spectrometry and surface profilometry to determine the densities from which we extract that $\rho_{\mathrm{WO}_{3}} \approx \rho_{\mathrm{NiWO}_{4}} \approx 1.4 \rho_{\mathrm{NiO}}{ }^{17,18}$ The film densities are in the $65 \pm 10 \%$ range as compared with bulk values. Table I lists the volume fractions of $\mathrm{NiWO}_{4}$ and $\mathrm{NiO}$ for the two structural models.

\section{B. Data}

Figure 5 compares data on $\varepsilon_{1}(E)$ and $\varepsilon_{2}(E)$ at $0.62 \leq E$ $\leq 5.62 \mathrm{eV}$ for $\mathrm{Ni}_{x} \mathrm{~W}_{1-x}$ oxide films with five compositions in the $0.05 \leq x \leq 0.43$ range. Data obtained from ellipsometry are compared with computations based on the Bruggeman effective medium theory of Eq. (3) and the volume fractions pertinent to the $\mathrm{WO}_{3}-\mathrm{NiWO}_{4}$ and the $\mathrm{WO}_{3}-\mathrm{NiO}$ structural models and given in Table I. At the lowest Ni content, with $x \approx 0.05$, the calculated results for the two nanostructural models almost coincide and are also in good agreement with the experimental values; no particular conclusions can be drawn from these data. Results for $x \approx 0.13$ are practically indistinguishable from those at $x \approx 0.05$ and are not shown. At higher $\mathrm{Ni}$ contents, however, it is found unambiguously that the $\mathrm{WO}_{3}-\mathrm{NiWO}_{4}$ nanostructural model represents the

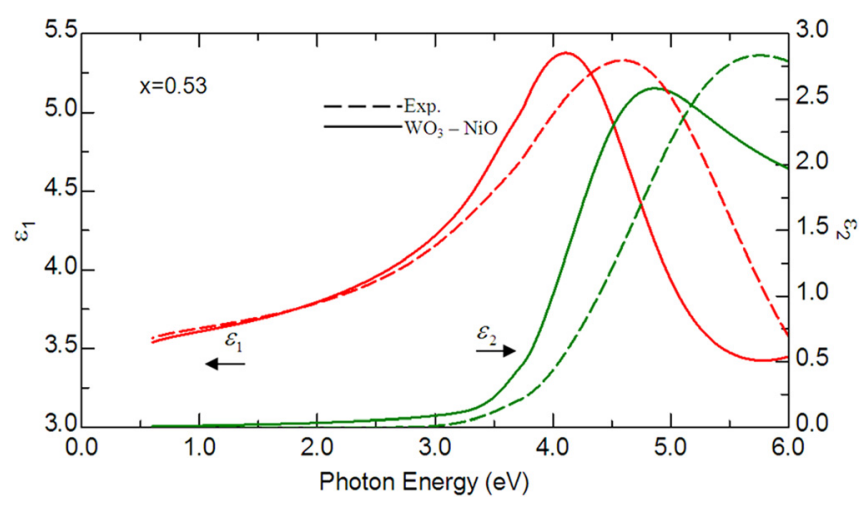

FIG. 6. Spectral complex dielectric function, $\varepsilon_{1}+i \varepsilon_{2}$ with pertinent vertical axes indicated by arrows, for a $\mathrm{Ni}_{x} \mathrm{~W}_{1-x}$ oxide film having the shown composition. Experimental data are shown together with calculations based on a nanostructural model, as shown in the figure. Experimental data were reported also in Fig. 4.

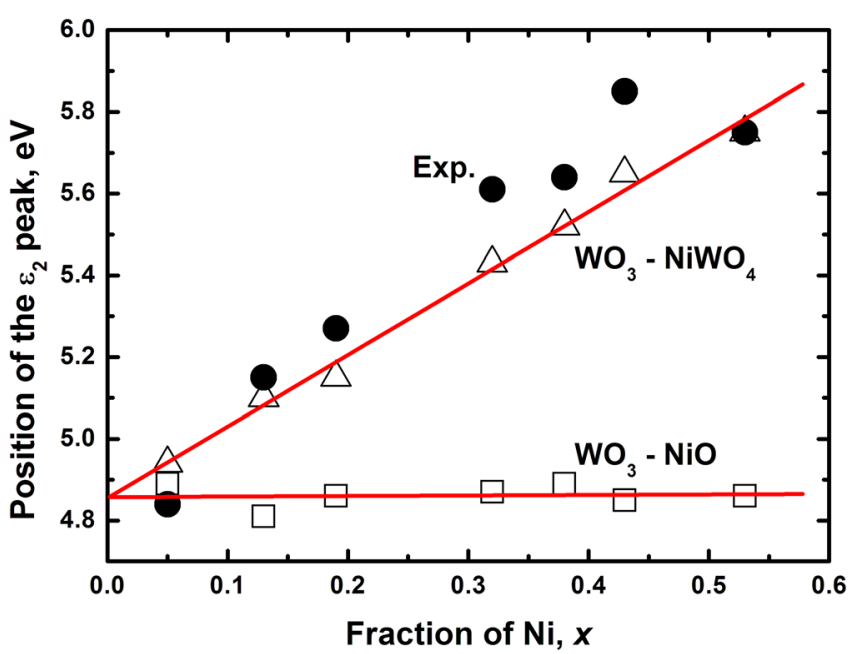

FIG. 7. Energy at the $\varepsilon_{2}$ peak for $\mathrm{Ni}_{x} \mathrm{~W}_{1-x}$ oxide film with the shown compositions $x$. Experimental data are shown together with calculations based on two nanostructural models, as shown in the figure. Lines were drawn for convenience to indicate theoretical results.

experimental data better than the $\mathrm{WO}_{3}-\mathrm{NiO}$ structural model does. For completeness, we performed a separate study of the sample with $x \approx 0.53$ and taken to represent $\mathrm{NiWO}_{4}$, which was compared with predictions from the $\mathrm{WO}_{3}-\mathrm{NiO}$ model. Figure 6 shows, expectedly, that this structural model fails.

Figures 5 and 6 test the qualitative agreement between theory and experiment. More quantitative assessments are possible by focusing on specific features in the data. Figure 7 shows the position of the $\varepsilon_{2}$ peak according to ellipsometry and as calculated from the two structural models. Clearly the data from the $\mathrm{WO}_{3}-\mathrm{NiWO}_{4}$ model are much superior to those of the $\mathrm{WO}_{3}-\mathrm{NiO}$ model, at least for $x>0.1$.

Attempts to fit the dielectric function of $\mathrm{Ni}_{x} \mathrm{~W}_{1-x}$ oxide films in the IR (cf. Fig. 3) to data obtained from computations based on the two nanostructural models were unsuccessful and did not lead to agreement except in the case of $x \approx 0.05$. It seems that the vibrational modes in the mixed oxide films cannot be reconciled with simple superpositions of those of the constituents but exhibit a more complex behavior.

\section{SUMMARY AND CONCLUSION}

Sputter deposited films of $\mathrm{Ni}_{x} \mathrm{~W}_{1-x}$ oxide with $0.05 \leq x$ $\leq 0.53$, having well documented electrochromism, ${ }^{6}$ were investigated by spectroscopic ellipsometry. Accurate data on the dielectric function were extracted in the 0.062 to $5.62 \mathrm{eV}$ range of photon energy. The results for 0.62 to $5.62 \mathrm{eV}$ were compared with computations based on the Bruggeman effective medium theory applied to two structural models: one describing a random mixture of structural entities characterized by the dielectric functions of $\mathrm{WO}_{3}$ and $\mathrm{NiWO}_{4}$ and the other representing a random mixture of $\mathrm{WO}_{3}$ and $\mathrm{NiO}$. Tungsten and nickel oxides have peaks in $\varepsilon_{2}$ at about $4.8 \mathrm{eV}$, and no peak shift is predicted for a mixture of them. This is contrary to our data which show a consistent peak shift towards the peak position of $\mathrm{NiWO}_{4}$. The different electronic properties of $\mathrm{NiWO}_{4}$ might be related to different chemical 
bonding, which is also consistent with a small shift of the infrared absorption peak in Fig. 3. Our analysis showed clearly that only the $\mathrm{WO}_{3}-\mathrm{NiWO}_{4}$ model was consistent with the experimental data. Hence, the films are composed of nanosized tungsten oxide and nickel tungstate. This result is in excellent agreement with our earlier investigation on the nanostructure of $\mathrm{Ni}_{x} \mathrm{~W}_{1-x}$ oxide films, ${ }^{17}$ where the information was inferred from Raman spectroscopy, x-ray photoelectron spectroscopy, and x-ray diffraction.

\section{ACKNOWLEDGMENTS}

This work was supported by grants from the Swedish Research Council for Environment, Agricultural Sciences and Spatial Planning (FORMAS) and the Swedish Research Council for Natural and Engineering Sciences (VR). One of us (I.V.) was supported by the European Research Council under the European Community's Seventh Framework Program (FP7/2007-2013)/ERC Grant Agreement No. 267234 (GRINDOOR) for work at Uppsala University. The Knut and Alice Wallenberg Foundation is acknowledged for financial support to instrumentation. One of us (S.V.G) was supported by Clear-up (Clean and Resource Efficient Buildings for Real Life), which is an integrated project funded by the European Community's Seventh Framework Programme (FP7/2007-2013) under Grant Agreement No. 211948.

${ }^{1}$ C. G. Granqvist, Handbook of Inorganic Electrochromic Materials (Elsevier, Amsterdam, The Netherlands, 1995).

${ }^{2}$ C. G. Granqvist, Sol. Energy Mater. Sol. Cells 99, 1 (2012).

${ }^{3}$ G. B. Smith and C. G. Granqvist, Green Nanotechnology: Solutions for Sustainability and Energy in the Built Environment (CRC, Boca Raton, FL, 2010).

${ }^{4}$ G. A. Niklasson and C. G. Granqvist, J. Mater. Chem. 17, 127 (2007).

${ }^{5}$ P. K. Shen, J. Syed-Bokhari, and A. C. C. Tseung, J. Electrochem. Soc. 138, 2778 (1991).
${ }^{6}$ S. V. Green, E. Pehlivan, C. G. Granqvist, and G. A. Niklasson, Sol. Energy Mater. Sol. Cells 99, 339 (2012).

${ }^{7}$ S.-H. Lee and S.-K. Joo, Sol. Energy Mater. Sol. Cells 39, 155 (1995).

${ }^{8}$ S.-H. Lee, Y.-S. Park, and S.-K. Joo, Solid State Ionics 109, 303 (1998).

${ }^{9}$ P. M. S. Monk, S. P. Akhtar, J. Boutevin, and J. R. Duffield, Electrochim. Acta 46, 2091 (2001).

${ }^{10}$ N. Penin, A. Rougier, L. Laffont, P. Poizot, and J.-M. Tarascon, Sol. Energy Mater. Sol. Cells 90, 442 (2006).

${ }^{11}$ D. Gillaspie, A. Norman, C. E. Tracy, J. R. Pitts, S. H. Lee, and A. Dillon, J. Electrochem. Soc. 157, H328 (2010).

${ }^{12}$ D. T. Gillaspie, R. C. Tenant, and A. C. Dillon, J. Mater. Chem. 20, 9585 (2010).

${ }^{13}$ A. Kuzmin, J. Purans, R. Kalendarev, D. Pailharey, and Y. Mathey, Electrochim. Acta 46, 2233 (2001).

${ }^{14} \mathrm{~S}$. Green, J. Backholm, P. Georén, C. G. Granqvist, and G. A. Niklasson, Sol. Energy Mater. Sol. Cells 93, 2050 (2009).

${ }^{15}$ I. Valyukh, S. Green, H. Arwin, G. A. Niklasson, E. Wäckelgård, and C. G. Granqvist, Sol. Energy Mater. Sol. Cells 94, 724 (2010).

${ }^{16}$ I. Valyukh, S. V. Green, C. G. Granqvist, G. A. Niklasson, S. Valyukh, and H. Arwin, Thin Solid Films 519, 2914 (2011).

${ }^{17}$ S. V. Green, A. Kuzmin, J. Purans, C. G. Granqvist, and G. A. Niklasson, Thin Solid Films 519, 2062 (2011).

${ }^{18}$ Handbook of Ellipsometry, edited by H. G. Tompkins and E. A. Irene (William Andrew, New York, 2005).

${ }^{19}$ C. M. Herzinger, B. Johs, W. A. McGahan, J. A. Woollam, and W. Paulson, J. Appl. Phys. 83, 3323 (1998).

${ }^{20}$ J. Tauc, R. Grigorovici, and A. Vancu, Phys. Status Solidi B 15, 627 (1966).

${ }^{21}$ J. A. Woollam Co., Inc., Guide to Using WVASE32 (WexTech Systems, 2010).

${ }^{22}$ S. Gou, H. Arwin, S. N. Jacobsen, K. Järrendahl, and U. Helmersson, J. Appl. Phys. 77, 5369 (1995).

${ }^{23}$ J. Koh, Y. Lu, C. R. Wronski, Y. Kuang, R. W. Collins, T. T. Tsong, and Y. E. Strausser, Appl. Phys. Lett. 69, 1297 (1996).

${ }^{24}$ R. Landauer, AIP Conf. Proc. 40, 2 (1978).

${ }^{25}$ D. E. Aspnes, Thin Solid Films 89, 249 (1982).

${ }^{26}$ G. A. Niklasson, in Materials Science for Solar Energy Conversion Systems, edited by C. G. Granqvist (Pergamon, Oxford, UK, 1991), pp. 7-43.

${ }^{27}$ G. A. Niklasson, C. G. Granqvist, and O. Hunderi, Appl. Opt. 20, 26 (1981).

${ }^{28}$ D. A. G. Bruggeman, Annal. der Physik 5, 636 (1935).

${ }^{29}$ J. C. Maxwell-Garnett, Philos. Trans. R. Soc. London, Ser. A, 203, 385 (1904); 205, 237 (1906) 